\title{
Zur Kenntnis der Cholsäure.
}

IV. Mitteilung.

Von

Martin Schenck.

(Aus dem pharmazeutisch-chemischen Institut der Universität Marburg).

(Der Redaktion zugegangen am 31. Januar 1914.)

Über Oximumlagerung bei Cholsäureabkömmlingen.

Unter den Oxydationsprodukten der Cholsäure bezw. der ihr nahestehenden anderen Gallensäuren (Choleinsäure, Desoxycholsäure) finden sich bekanntlich verschiedene Verbindungen, welche Carbonyl-Sauerstoff enthalten und demgemäß mit Hydroxylamin zu reagieren befähigt sind. Am meisten untersucht sind von diesen Verbindungen die bei der Oxydation der Cholsäure entstehenden Körper: Dehydrocholsäure, $\mathrm{C}_{24} \mathrm{H}_{34} \mathrm{O}_{5}$, und Biliansäure, $\mathrm{C}_{24} \mathrm{H}_{34} \mathrm{O}_{8}$. Die Dehydrocholsäure enthält außer einer Carboxylgruppe drei Carbonylgruppen und liefert dementsprechend mit Hydroxylamin ein Trioxim. Von den drei Carbonylgruppen sollen zwei Aldehydgruppen, die dritte eine Ketongruppe sein; die letztere darf wohl als ringförmig gebunden angesehen werden, denn aus dem Molekül der Dehydrocholsäure wird selbst bei verhältnismäßig energischer 0xydation zunächst noch kein Kohlenstoff abgespalten, was zu erwarten wäre, wenn die Ketongruppe in offener Kette sich befände. Wie bereits in der vorigen Mitteilung ${ }^{1}$ ) betont wurde, sind aber vielleicht auch die beiden als Aldehydgruppen angenommenen Carbonyle ringförmig gebundene Ketongruppen. Die Biliansäure, die bei weitergehender Oxydation der Cholsäure aus zunächst entstandener Dehydrocholsäure gebildet wird, enthält außer drei Carboxylgruppen zwei allgemein als

1) Diese Zeitschrift, Bd. 87. S. $59 \mathrm{ff}$. 
Ketongruppen angenommene Carbonyle und vermag aus diesem Grunde mit Hydroxylamin unter Bildung eines Dioxims in Reaktion zu treten. Auch hier sind die Ketongruppen wahrscheinlich ringförmig gebunden, denn auch die Biliansäure liefert bei ziemlich energischer Oxydation zunächst kein kohlenstoffärmeres Produkt (vgl. die vorige Mitteilung ${ }^{1}$ ) über die Formel der Ciliansäure, des Oxydationsproduktes der Biliansäure).

Es lag nahe zu versuchen, wie sich die Oxime der genannten Cholsäureabkömmlinge gegenüber solchen Reagenzien verhalten würden, welche die Beckmannsche Umlagerung bewirken. Wir wissen durch die Untersuchungen von W alla ch, ${ }^{2}$ ) daß sich die Oxime gesättigter zyklischer Ketone glatt analog dem Beckmannschen Schema in Isoxime (Lactame) umlagern lassen, die dann ihrerseits zu Aminosäuren aufgespalten werden können. So geht z. B. Pentanonoxim über in $\alpha$-Piperidon, daszu ठ-Aminovaleriansäure aufgespalten werden kann(l.c.S.181):

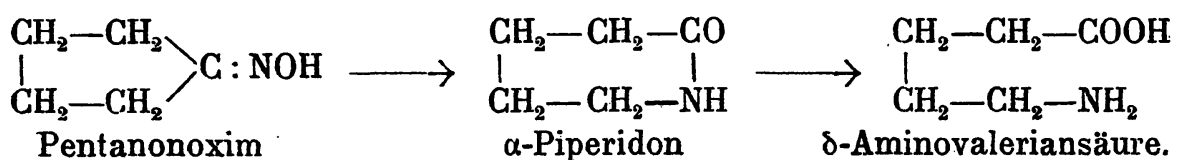

Ähnliche Umlagerungen waren bei den Oximen der erwähnten Cholsäurederivate zu erwarten - die Cholsäure dürfte ja zu den gesättigten zyklischen Verbindungen zu zählen sein und haben sich in der Tat beim Trioxim der Dehydrocholsäure und beim Dioxim der Biliansäure realisieren lassen. Darüber soll im folgenden berichtet werden. Ich beabsichtige, die erhaltenen Umlagerungsprodukte aufzuspalten und die hierbei eventuell entstehenden Aminosäuren weiter abzubauen. Vielleicht gelingt es auf diese Weise, neue charakteristische Abbauprodukte der Cholsäure zu gewinnen. Die Aufspaltungsversuche werden voraussichtlich auch geeignet sein zur Entscheidung der Frage, ob die von mir gemachte Annahme, daß alle drei Carbonyle der Dehydrocholsäure Ketongruppen sind, zu Recht besteht. Die Zusammensetzung des unten beschriebenen Umlagerungsproduktes des Dehydrocholsäuretrioxims scheint sehr für diese

1) Diese Zeitschrift, Bd. 87, S. こั9 ff.

2) Ann. d. Chem., Bd. 312, S. 171. 
Annahme zu sprechen. Auch sollen andere von der Cholsäure bezw. Choleinsäure und Desoxycholsäure sich ableitende Carbonylsäuren in den Bereich der Untersuchung gezogen werden.

\section{Umlagerung des Trioxims der Dehydrocholsäure: Isotrioxim der Dehydrocholsäure.}

Das Trioxim der Dehydrocholsäure wurde aus reiner Dehydrocholsäure nach den Angaben von Mylius ${ }^{1}$ ) bereitet. Der Zersetzungspunkt lag bei 273 bis $274^{\circ}$; Mylius gibt an, daß das Oxim sich jenseits $270^{\circ}$ zersetzt. Die Analyse der Substanz lieferte mit der Theorie übereinstimmende Werte:

$0,1980 \mathrm{~g}: 0,4696 \mathrm{~g} \mathrm{CO}_{2}$ und $0,1519 \mathrm{~g} \mathrm{H}_{2} \mathrm{O}$; gef. $64,68 \% \mathrm{C}$ und $8,58 \% \mathrm{H}$; $0,1759 \mathrm{~g}: 14 \mathrm{ccm} \mathrm{N}$ bei $14^{\circ}$ und $744,2 \mathrm{~mm} \mathrm{Hg}$; gef. $9,27 \% \mathrm{~N}$.

Ber. für $\mathrm{C}_{34} \mathrm{H}_{37} \mathrm{~N}_{3} \mathrm{O}_{5}: 64,37 \% \mathrm{C}, 8,33 \% \mathrm{H}$ und $9,41 \% \mathrm{~N}$.

Zur Umlagerung des Oxims verwendete ich ebenso wie Wallach (l. c.) mit wenig Wasser verdünnte reine Schwefelsäure und zwar wurden im Meßzylinder $20 \mathrm{ccm}$ Wasser unter Abkühlen mit konzentrierter Schwefelsäure vermischt und die Mischung mit konzentrierter $\mathrm{H}_{2} \mathrm{SO}_{4}$ auf $120 \mathrm{ccm}$ aufgefüllt. Es dürfte diese Mischung etwa einer $90 \%$ igen Schwefelsäure entsprechen. Man darf nun aber die Lösung des Trioxims in dieser starken Schwefelsäure nicht - selbst nicht ganz kurze Zeit - zum Aufwallen erhitzen, wie es Wallach bei seinen Oximen tun durfte, es würde sonst eine tiefgreifende Zersetzung des Trioxims unter intensiver Schwärzung der Flüssigkeit eintreten; man muß vielmehr Dampfbadtemperatur anwenden. Allerdings läßt sich auch so eine gewisse Braunfärbung des Reaktionsgemisches nicht vermeiden, die indessen in der gleich zu erwähnenden Weise beseitigt werden kann.

Die Umlagerung des Trioxims gestaltet sich also folgendermaßen: $1 \mathrm{~g}$ Dehydrocholsäure-Trioxim werden mit $12 \mathrm{ccm}$ der starken Schwefelsäure in einem kleinen Kölbchen 15-20 Minuten lang auf dem Dampfbad erhitzt, die Substanz geht dabei alsbald in Lösung und die Lösung färbt sich etwas bräunlich. Nach dem Abkühlen wird die Flüssigkeit in wenig Wasser, das

1) Ber., Bd. 19, S. 2007. 
durch eingetragene Eisstückchen gekühlt ist, gegossen, und die so erzielte verdünnte Lösung unter Eiskühlung mit Natronlauge schwach alkalisch gemacht. Dann setzt man vorsichtig tropfenweise verdünnte Essigsäure $\mathrm{zu}$, wodurch zunächst bräunlich gefärbte Flocken ausgefällt werden. Von diesen Flocken wird abgesaugt und aus dem Filtrat durch weiteren vorsichtigen $\mathrm{Zu}$ satz von Essigsäure das Reaktionsprodukt vollständig ausgefällt. Bei richtiger Arbeitsweise erhält man das letztere so in Form einer schneeweißen Masse. Diese wird abgesaugt und aus stark verdünntem Alkohol (20 Teile Alkohol, 80 Teile Wasser) umkrystallisiert. Auch aus reinem Wasser, in welchem das Produkt sehr schwer löslich ist, läßt es sich umkrystallisieren. Seine krystallinische Beschaffenheit ist unter dem Mikroskop zu erkennen: es bildet Kugeln, die am Rande Spitzen und Zacken zeigen und dadurch ihre Entstehungsweise dokumentieren; mitunter beobachtet man auch ganz deutlich sphärische Aggregate von Nadeln, manchmal auch isolierte Krystalle, die eigentümlich zackig, wie zerhackt aussehen. Das Umlagerungsprodukt unterscheidet sich sofort von dem in Alkohol sehr schwer löslichen Trioxim durch seine leichte Löslichkeit in Alkohol, ferner zersetzt sich das Isotrioxim bei erheblich niedrigerer Temperatur als das Ausgangsmaterial, allerdings ist der Zersetzungspunkt der Iso-Verbindung wenig scharf, sie beginnt bei etwa $187^{\circ}$ sich zu bräunen und schäumt bei ca. $210^{\circ}$ auf. Die Analyse der bei $100^{\circ}$ getrockneten Substanz ergab, daß tatsächlich eine Verbindung von der gleichen Zusammensetzung, wie sie das Trioxim aufweist, vorlag:

$0,1486 \mathrm{~g}: 0,3486 \mathrm{~g} \mathrm{CO}_{2}$ und $0,1098 \mathrm{~g} \mathrm{H}_{2} \mathrm{O}$; gef. $63,98 \% \mathrm{C}$ und $8,27 \% \mathrm{H}$; $0,2096 \mathrm{~g}: 17,6 \mathrm{ccm} \mathrm{N}$ bei $15,5^{\circ}$ und $744 \mathrm{~mm} \mathrm{Hg}$; gef. $9,73 \% \mathrm{~N}$; $0,1529 \mathrm{~g}: 12,4,>, 12,5^{\circ}=754,4,>;>9,63 \%$, Ber. für $\mathrm{C}_{24} \mathrm{H}_{37} \mathrm{~N}_{8} \mathrm{O}_{5}: 64,37 \% \mathrm{C}, 8,33 \% \mathrm{H}$ und $9,41 \% \mathrm{~N}$.

Sehr geeignet zur Unterscheidung des Isotrioxims von dem Trioxim ist das folgende charakteristische Verhalten der beiden Verbindungen. Beide Körper lösen sich leicht in Salzsäure auf. Kocht man nun die salzsaure Lösung des Trioxims einige Zeit, so scheidet sich in Salzsäure sehr schwer lösliche Dehydrocholsäure aus; es hat eben eine Abspaltung der Oximgruppen 
unter Regenerierung der ursprünglichen Carbonylsäure stattgefunden, eine Erscheinung, die bereits Mylius (l. c.) beobachtet hatte. Erhitzt man dagegen die salzsaure Lösung des Isotrioxims unter den gleichen Bedingungen, so bleibt sie auch beim $\mathrm{Ab}-$ kühlen vollständig klar; es ist offenbar eine Aufspaltung des Isoxims eingetreten, aber das gebildete Produkt (Aminosäure) ist infolge der Anwesenheit der basischen Gruppen ebenso wie das Isoxim in Salzsäure löslich.

Umlagerung des Dioxims der Biliansäure: Isodioxim der Biliansäure.

Die Bereitung des Dioxims der Biliansäure(Isonitrosobiliansäure) geschah nach der Vorschrift von Mylius, ${ }^{1}$ ) nur bin ich insofern etwas von der Myliusschen Arbeitsweise abgewichen, als ich mehr Natronlauge genommen habe. Mylius gibt nämlich an, daß es beim Erwärmen des Reaktionsgemisches (Biliansäure + Natronlauge + Hydroxylaminchlorhydrat) zu einer Ausscheidung weißer Krystallblättchen, die ein saures Natriumsalz des Biliansäuredioxims darstellen, kommt, und daß die Hauptmenge des gesuchten Dioxims aus dem Filtrat von dem sauren Natriumsalz durch vorsichtigen Zusatz verdünnter Salzsäure niedergeschlagen werden kann. Um die Ausscheidung des sauren Natriumsalzes zu vermeiden, habe ich die Menge der Natronlauge so bemessen, daß sie der Biliansäure und der Salzsäure des Hydroxylaminchlorhydrats äquivalent war. Unter diesen Bedingungen scheidet sich beim Erwärmen der Lösung auf dem Dampfbade nichts aus und auch beim Abkühlen bleibt die Flüssigkeit vollständig klar. Das Dioxim wurde dann durch vorsichtigen Zusatz von Essigsäure abgeschieden und aus verdünntem Alkohol umkrystallisiert. Es zersetzte sich bei $266^{\circ}$, Pregl ${ }^{2}$ ) gibt an, daß sich die Isonitrosobiliansäure bei $270^{\circ} \mathrm{zu}$ zersetzen beginnt. Eine Stickstoffbestimmung gab einen mit der Theorie übereinstimmenden $\mathrm{N}-\mathrm{Wert}$ :

0,2062 g Subst.: $10,3 \mathrm{ccm} \mathrm{N}$ bei $8^{\circ}$ und $747 \mathrm{~mm} \mathrm{Hg}$; gef. $5,97 \% \mathrm{~N}$. Ber. für $\mathrm{C}_{24} \mathrm{H}_{36} \mathrm{~N}_{2} \mathrm{O}_{8}: 5,85 \% \mathrm{~N}$.

1) Ber., Bd. 20, S. 1984.

2) Monatsh. f. Chem., Bd. 24, S. 56. 
Die Umlagerung des Dioxims geschah in ganz der gleichen Weise wie beim Dehydrocholsäuretrioxim. $1 \mathrm{~g}$ Dioxim wurde wieder mit $12 \mathrm{ccm}$ der starken Schwefelsäure in einem kleinen Kölbchen 15 Minuten lang auf dem Dampfbad erhitzt, die Substanz ging dabei alsbald in Lösung und die Lösung färbte sich nur ganz schwach, bräunlich, sodaß auf diese Färbung keine Rücksicht genommen zu werden brauchte. Zur Isolierung des Biliansäureisodioxims muß man bei größerer Konzentration der Lösung arbeiten, als dies beim Dehydrocholsäureisotrioxim geschehen kann, weil aus verdünnter Lösung das Isodioxim sich nur unvollständig ausscheidet. Demgemäß wurde das Reaktionsgemisch nach dem Erkalten auf Eis gegossen und die erzielte Lösung unter Eiskühlung mit starker Natronlauge (von ca. $22 \%$ ) nach und nach versetzt; dabei kommt es vorübergehend zu einer Ausscheidung des Isodioxims, das aber bei gerade hinreichendem Zusatz der Lauge wieder vollständig in Lösung geht. Nun wurde vorsichtig tropfenweise verdünnte (10\%ige) Schwefelsäure zugegeben und das Isodioxim so möglichst vollständig niedergeschlagen. Nach mebrstündigem Stehen, wobei es zu einer reichlichen Ausscheidung von Natriumsulfat kommt, wurde abgesaugt und der auf dem Filter verbleibende Rückstand zunächst mit kaltem Wasser behandelt, um das Natriumsulfat zu beseitigen, und hierauf aus heißem Wasser umkrystallisiert. Es resultiert das Isodioxim dabei in Gestalt von mikroskopisch kleinen, schỏnen Nadeln, die meist sternförmig gruppiert erscheinen; mitunter, bei langsamem Auskrystallisieren, beobachtet man unter dem Mikroskop etwas größere Krystalle, die die Form von langgestreckten Rechtecken haben, die längeren Seiten erscheinen dabei schwach gewölbt, sodaß die Breite des Krystalls von der Mitte nach den Enden zu etwas abnimmt. Das Isodioxim beginnt bei ca. $250^{\circ}$ sich schwach zu bräunen und schäumt bei ca. $258^{\circ}$ unter Zersetzung auf. Die Analyse des bei $100^{\circ}$ getrockneten Körpers ergab, daß eine mit dem Ausgangsmaterial gleich zusammengesetzte Verbindung vorlag:

$0,1511 \mathrm{~g}$ Subst. : 0,3325 g CO, u. 0,1013 $\mathrm{g} \mathrm{H}_{2} \mathrm{O}$; gef. $60,02 \% \mathrm{C}$ u. 7,50\% $\mathrm{H}$; $0,1996>?: 9,5 \mathrm{ccm} \mathrm{N}$ bei $14,5^{\circ}$ und $747,1 \mathrm{~mm} \mathrm{Hg}$; gef. $5,56 \% \mathrm{~N}$; $0,2053,>: 9,8>>>15^{\circ}, 747,1>>; 5,56 \%$, Ber. für $\mathrm{C}_{24} \mathrm{H}_{36} \mathrm{~N}_{2} \mathrm{O}_{8}: 59,96 \% \mathrm{C}, 7,55 \% \mathrm{H}$ und $5,85 \% \mathrm{~N}$. 
Das Isodioxim ist in ( $96 \%$ igem) Alkohol ziemlich schwer löslich, wenn auch offenbar etwas leichter als das Dioxim. Abgesehen von dem - allerdings nur etwa $10^{\circ}$ - niedriger liegenden Zersetzungspunkt unterscheidet sich der Isokörper von dem Dioxim wesentlich durch die Krystallform (das in Wasser sehr schwer lösliche Biliansäuredioxim bildet beim Umkrystallisieren aus Wasser unter dem Mikroskop zu erkennende, ganz kleine, sechsseitige Täfelchen, die nicht die entfernteste Ähnlichkeit mit den eben beschriebenen Nadeln des Isodioxims besitzen). Am meisten geeignet zur Unterscheidung der beiden isomeren Verbindungen ist aber wieder das Verhalten gegen siedende Säure. Beide Körper lösen sich in kalter (etwa 25\% iger). Salzsäure auf; erhitzt man einige Zeit, so scheidet sich aus der Lösung des Dioxims, namentlich beim Erkalten, die schwer lösliche Biliansäure aus, die Lösung des Isodioxims bleibt dagegen, auch beim Abkühlen, vollständig klar. Die Gründe für dieses verschiedene Verhalten sind die gleichen, wie sie oben für Trioxim und Isotrioxim der Dehydrocholsäure angegeben wurden.

Bei der Beschaffung von Ausgangs-Rohmaterial bin ich von der Gräfin Louise Bose-Stiftung in sehr dankenswerter Weise durch eine Geldbeihilfe unterstützt worden. 